\title{
Discovery and investigation of the GAIT translational control system
}

\author{
PAUL L. FOX \\ Department of Cellular and Molecular Medicine, Cleveland Clinic Lerner Research Institute, Cleveland, Ohio 44195, USA
}

My laboratory has been studying the GAIT translational control pathway for almost exactly 20 years. We stumbled into the translation field entirely by accident, without forethought, and with little knowledge or experience. Barsan Mazumder and Chinmay Mukhopadhyay, two fellows in my lab investigating ceruloplasmin (Cp) expression, observed that interferon (IFN)- $\gamma$ induced monocytic cell expression of $\mathrm{Cp}$, a secreted acute phase protein, as well as its transcript. The inducible level of $C P$ mRNA and Cp protein in the conditioned medium both flattened after about $12 \mathrm{~h}$. At first glance this was not a surprising finding. But we soon realized the result was, in fact, unusual and suggested a mechanism beyond simple transcription and translation. Because Cp is a highly stable, secreted protein, we expected it to accumulate continuously in the conditioned medium in the presence of a constant amount of $C P$ mRNA. We were astute enough to recognize the interesting disparity, but not astute enough to pursue it. We mentioned this apparent discrepancy in the discussion of our submitted manuscript and suggested that it might be explained by delayed inhibition of translation of $C p$ mRNA. Fortunately, we had a more astute reviewer who asked us to provide experimental evidence for this hypothesis. The request led to a key experiment that changed the direction of my laboratory for the next 20 years (and is still ongoing): Metabolic labeling with ${ }^{35} \mathrm{~S}$-methionine unambiguously showed that despite continued, robust expression of $C p$ mRNA, synthesis of Cp ceased completely after $24 \mathrm{~h}$. We were faced with starting a research program in transcript-selective translational control!

Not knowing whether or how to pursue translational control mechanisms, I read a review by Nancy Standart and Richard Jackson, published in Biochimie in 1994. I found the article fascinating and inspiring, with an insight on nearly every page, and my decision was made. Sometime later I told Richard about his important influence on my research direction-he quipped that he thought I was the only person to have read that review. We soon found that the $3^{\prime}$-untranslated region (UTR) of Cp mRNA was essential for the observed

\footnotetext{
Corresponding author: foxp@ccf.org

Article and publication date are at http://www.rnajournal.org/cgi/doi/ 10.1261/rna.050187.115. Freely available online through the RNA Open Access option.
}

translational silencing. This finding was not completely unexpected given earlier studies by Matthias Hentze and others showing that this region was at least as important for translational control as the $5^{\prime}$ UTR. Nonetheless, we were puzzled by the mechanism by which the $3^{\prime}$ UTR could influence translation initiation at the distant $5^{\prime}$ UTR. In a fruitful collaboration with Nahum Sonenberg, we showed that end-to-end closure of $C P$ mRNA was essential for translational repression. Previously, others had shown that mRNA circularization contributed to translation efficiency, but our results uniquely showed that transcript circularization was essential for GAIT-mediated translational control, and in fact decreased translation efficiency of the target mRNA. This result was very satisfying because it revealed a simple answer to the conundrum raised by translational control by the $3^{\prime}$ UTRthe $3^{\prime}$ and $5^{\prime}$ UTRs are proximate.

We recognized the urgency of identifying the specific cisand trans-acting constituents driving translational control. Prabha Sampath, a new graduate student in the lab, tackled the identification of the $3^{\prime}$-UTR element in Cp mRNA. By a comprehensive deletion and mutation analysis, in 2003 she described a minimal, 29-nt split stem-loop element that could drive translational silencing of a heterologous transcript. We termed this RNA element the GAIT, or interferon-gamma activated inhibitor of translation, element. Barsan and Prabha then joined forces to seek the trans-acting factor(s) that bound the GAIT element and silenced translation. Taking advantage of the genetic yeast 3-hybrid approach, Barsan identified ribosomal protein (RP) L13a as a critical GAIT component. Taking a proteomic approach, and using her new-found $C p$ GAIT element as bait, Prabha identified three other GAIT complex constituents by mass spectrometry-glutamyl-prolyl tRNA synthetase (EPRS), NS1-associated protein 1 (NSAP1), and glyceraldehyde-3phosphate dehydrogenase (GAPDH). To validate these candidates (and invalidate others) we begged and borrowed (and occasionally purchased) a host of antibodies. I called Paul Schimmel, whom I'd never met, to request the antiEPRS antibody. He didn't have the reagent, but suggested

(C) 2015 Fox This article, published in $R N A$, is available under a Creative Commons License (Attribution-NonCommercial 4.0 International), as described at http://creativecommons.org/licenses/by-nc/4.0/. 
I could get it from Sunghoon Kim in Seoul, Korea. He told me that we had landed smack in the middle of the most important, emerging area in the synthetase field, i.e., noncanonical functions unrelated to tRNA ligation. I was even more surprised when Paul suggested that his lab likes to know everything going on in the synthetase field, and he invited me to give a talk at Scripps the very next week! I declined, at least until we confirmed EPRS as an authentic GAIT constituent, but Paul has been an enthusiastic proponent of our efforts ever since. Remarkably, our two-pronged strategy worked perfectly as Barsan and Prabha discovered complementary proteins not detected by the other. All four assembled into a functional GAIT element-binding, inhibitory complex. Later, Jie Jia and Abul Arif showed, by in vitro reconstitution experiments with purified proteins, that the four constituents were not only necessary, but also sufficient to induce translational silencing.

Our early studies revealed a rather unexpected discovery, namely, two GAIT complex proteins were inducibly phosphorylated and released from their parental complexesRP L13a from the large ribosomal subunit and EPRS from the tRNA multisynthetase complex (MSC). To our knowledge, stimulus-inducible release of proteins from "stable" intracellular machines had not been reported. We proposed the "depot hypothesis" that generalized our findings to suggest that other "stable" complexes can release components to perform "noncanonical" functions unrelated to their original activity. These results motivated several investigators of tRNA synthetases who subsequently showed that other constituents were inducibly phosphorylated and released from the MSC to perform potentially important cellular functions. Our findings was less enthusiastically received by the ribosome community that focuses primarily on ribosome assembly, structure, and catalysis; a constituent dropping off the ribosome was of much less interest, particularly since it didn't influence overall protein synthesis. When I described these early studies at a Cold Spring Harbor Translational Control meeting in the mid-nineties, Peter Sarnow of Stanford surmised that we had landed on the tip of a very large iceberg, and it will be very exciting to explore its hidden regions. I was very glad to hear this from Peter since I was suspecting (and hoping) the same. During the following decade we have been exploring the distal reaches of the GAIT "iceberg" and will here relate a few of the more intriguing findings, some rather far afield from translational control.

Follow-up studies showed that both stimulus-releasable proteins served critical GAIT functions. After starting his own lab, Barsan Mazumder, in a collaborative effort with us, Anton Komar, and Bill Merrick, showed that RP L13a interacted with eIF4G, and was the direct inhibitor of translation-initiation. The protein that recognized the GAIT element was not immediately obvious since all four GAIT constituents are known RNA-binding proteins. An RNAshift experiment by Jie Jia using purified proteins conclusively showed that EPRS was the sole direct GAIT element-bind- ing protein, and thus became a major focus of our efforts. EPRS contains two catalytic tRNA synthetases in one polypeptide chain, and as such is the only bifunctional synthetase. Jie soon discovered that the linker connecting the synthetases was entirely responsible for GAIT element RNA binding. The human EPRS linker contains three helix-turn-helix WHEP domains. The first two bound the RNA element, whereas the second overlapping pair bound the other three GAIT proteins. Abul Arif then showed that the two phosphorylation events critical for EPRS activation and release from the MSC also occurred in the linker region. These results and others from the Schimmel group at Scripps led to the concept, formalized by Min Guo and colleagues, that the appended domains in eukaryotic synthetases, absent in their bacterial homologues, are responsible for the diverse noncanonical functions. These observations piqued the curiosity of Partho Sarothi Ray who began to sequence his way back in evolutionary time to find the organism in which the ERS and PRS synthetases first became joined. In a surprising stop along the way, Partho showed that the stinging sea anemone, Nematostella vectensis, contained three alternatively-spliced linked forms EPRS containing either one or two WHEP domains. A 1-WHEP domain linker bound human tRNA with high affinity and a 2-WHEP domain linker bound the GAIT element, suggesting that the WHEP domains possibly originated to capture tRNA, thereby facilitating ligase activity, and later evolved the noncanonical functions. Recently, Partho identified the fusion event near the divergence of ichthyosporea and the emergence of filozoa almost a billion years ago, very near the emergence of animal-like unicellular organisms.

We were particularly interested in the target scope of the GAIT system, and soon identified and validated mRNAs encoding vascular endothelial growth factor A (VEGFA), death-associated protein kinase (DAPK) and zipper-interacting protein kinase $(Z I P K)$ as authentic GAIT targets. Rupak Mukopadhyay showed that both kinases were not only targets, but also formed a cascade that phosphorylated and activated RP L13a at Ser ${ }^{77}$, thereby activating the GAIT complex, and indicating the GAIT system is auto-regulated by a negativefeedback loop. Barsan Mazumder subsequently identified several chemokines and their receptors as GAIT targets. Clearly, the GAIT system was the hub of a decent-sized "post-transcriptional regulon," but we suspect that interesting new targets, and the overall scope, remain to be determined.

At about this time we made a puzzling but intriguing observation. As expected, we observed a near-linear correlation between VEGFA mRNA and VEGF-A protein in the cell lysate. However, this relationship fell apart at later times after IFN- $\gamma$ treatment, when translational silencing was in effect. During this period a small, constant "trickle" of synthesis of target proteins was observed that was independent of the expression of CP or VEGFA mRNA, revealing a hyperbolic or "saturable" relationship. We surmised that the feedback loop we had previously observed was responsible for the unusual system 
behavior, and teamed up with Jerry Saidel, an expert in mathematical modeling, to model the GAIT system. Unfortunately, no matter how we manipulated the system components and rate constants, we were unable to model the hyperbolic relationship. After a few years of occasional mulling over the problem, I suddenly realized that a small amount of a molecule that bound the GAIT element with high affinity, but didn't form the GAIT complex, might yield the desired result. Jerry and Alka Potdar added this putative component to their system of differential equations and-voila! —out popped a hyperbola. We soon realized that a fragment of EPRS that contained only the first two WHEP domains, or a structurally-related protein, might satisfy the required criteria-it would bind the GAIT element but not permit complex assembly. Peng Yao then showed that monocytic cells express an $\mathrm{N}$ terminal fragment of EPRS shown by mass spectrometry to contain only the first two WHEP domains and, indeed, satisfied the criteria. We guessed that the fragment, termed $\mathrm{EPRS}^{\mathrm{N} 1}$, might be a proteolytic product, but a detailed analysis revealed a far more interesting genetic mechanism. EPRS ${ }^{\mathrm{N} 1}$ is generated by alternative polyadenylation after "UA" in a Tyr-encoding UAU codon in the coding sequence of EPRS to generate a UAA stop codon and a truncated mRNA. To our knowledge, this was the first report of coding sequence polyadenylation generating a stable mRNA and protein; likely, the introduction of a new stop codon was critical since it prevented nonstop mRNA decay. Following our report, the Steinmetz group found almost thirty truncated yeast mRNAs in which a Tyr codon was similarly polyadenylated to generate a stop codon and truncated mRNA and protein, suggesting an evolutionarily conserved mechanism for proteome diversification. I remain surprised that there are not computational approaches (at least to my knowledge) that randomly "try out" new system components in the mathematical modeling of a multi-component system; this could have saved us a few years. Nonetheless, we learned an important lesson from this study and from our original discovery of the GAIT system: My former Cornell professor, Ephraim Racker, used to admonish us to "Never ignore the lag"; I would add, "Never ignore the plateau."

We were curious to know if pathophysiological conditions influence GAIT system activity, particularly with respect to the important angiogenic factor, VEGF-A. We knew that IFN- $\gamma$ inhibited VEGF-A expression, whereas, hypoxia stimulated its expression. We wondered which effector was dominant when combined. I mentioned this question to Greg Semenza who discovered the hypoxia-inducible factor 1 (HIF-1) transcription factor. He immediately answered "hypoxia." I asked how he knew, and he responded: "Hypoxia always wins." He was right in our case, too. Tackling this problem, Partho Ray discovered a hypoxia-responsive RNA switch in the VEGFA $3^{\prime}$ UTR that was responsible for the response. Immediately adjacent to the GAIT element there was a CA-rich binding site for hnRNP L (heterogeneous nuclear ribonucleoprotein). Following hypoxic treatment, hnRNP L binds the $3^{\prime}$ UTR and induces a conformational switch so that the GAIT element is unable to form, and translational silencing is prevented. Peng Yao later showed that hnRNP L was activated by phosphorylation and cytoplasmic re-localization, and also that it did not work alone. He elucidated the heterotrimeric HILDA (hypoxia-inducible hnRNP L-double stranded RNA-binding protein 76 [DRBP76]-hnRNP A2B1) complex that drives the conformational switch. Remarkably, the 3'-UTR switch contains a triad of adjacent elements; DRBP76 binds an AU-rich stem-loop that "flips" the RNA switch by disrupting the GAIT element, driving robust VEGFA mRNA translation.

A second pathophysiological agent we considered was oxidatively modified LDL (oxLDL) which can load cholesterol into macrophages, and is generally considered to be the very worst form of "bad cholesterol" contributing to atherosclerosis. Jie Jia showed that oxLDL completely inhibited GAIT system activity, thereby increasing expression of GAIT targets. Remarkably, oxLDL, in the presence of IFN- $\gamma$, induced the disappearance of essentially the entire cellular pool of RP L13a, without influencing other GAIT complex components or overall protein synthesis. OxLDL was shown to induce specific $S$-nitrosylation of GAPDH at $\mathrm{Cys}^{247}$ which prevented its binding to RP L13a leading to its rapid ubiquitination and proteasomal degradation. Rapid degradation of free RPs in the cytoplasm, and thus unprotected by the ribosome, is a mechanism for maintaining the 1:1 stoichiometry. We finally had the answer to a question unfailingly asked at every presentation of the GAIT system, i.e., "what does GAPDH do?" GAPDH acts as an RP L13a chaperone, stabilizing it when off the ribosome and perhaps in the GAIT complex as well. Jie went on to pursue the mechanism underlying GAPDH nitrosylation. He identified a novel, stimulusinducible nitrosylase complex consisting of the inflammatory myeloid proteins S100A8 and S100A9, as well as inducible nitric oxide synthase (iNOS). He showed that a conserved I/L-X-C- $\mathrm{X}_{2}-\mathrm{D} / \mathrm{E}$ motif was necessary and sufficient for target-selective $S$-nitrosylation of GAPDH, and other targets, by the complex. A primary sequence motif for $S$-nitrosylation had not been described previously, thus our work on regulation of translational control unexpectedly revealed a longsought parallel between $S$-nitrosylation and phosphorylation.

There remain many unexplored regions of the GAIT "iceberg," including mechanisms of release of EPRS and RP L13a from the parental complexes, upstream activation pathways, and mechanisms of assembly of the GAIT complex. Also lacking is detailed structural information on the complexes discovered during our exploration, i.e., the GAIT, HILDA, and S100A8/A9-iNOS, or on their interactions with targets. Moreover, any influence of microRNAs on GAIT system function has not been reported to date. A critical gap remains in our knowledge of the in vivo function(s) of the GAIT system. The Mazumder lab has begun to tackle the issue using myeloid-specific, L13a-deficient mice. Their findings on inflammation and atherosclerosis are consistent with an 
important anti-inflammatory function of RP L13a. However, L13a contributes to other processes, including ribosomal RNA methylation and cap-independent translation, and thus GAIT-independent functions of L13a might overshadow GAIT-dependent ones. We are taking a complementary approach in which loss- or gain-of-function mutations are introduced into the key phosphorylation sites in RP L13a and EPRS to prevent or activate the GAIT system in mice without disruption of other functions. The function of the GAIT system in human health, and its pathological dysregulation, is also of great interest to us. We are gratified that several of our findings have been extended by others, e.g., constituent release from the MSC and in-CDS polyadenylation, but we hope that other results from our studies will be found applicable to other systems. For example, are other ribosomal proteins inducible released? Does RNA circularization contribute to other translational control systems, including those regulated by microRNA? Are there proteindirected conformational switches in the $3^{\prime}$ UTRs of other transcripts?

The last decade has seen a resurgence of interest in $3^{\prime}$-untranslated regions (UTRs) of transcripts, primarily because they are frequent targets of microRNAs. But no less important, and possibly more significant quantitatively, is the targeting of these regions by trans-acting factors that regulate transcript translation (as well as stability). We are grateful that our findings on the GAIT system have motivated and instructed others, and we hope that others will enjoy their exploration of translational control systems as much as we have enjoyed ours. 

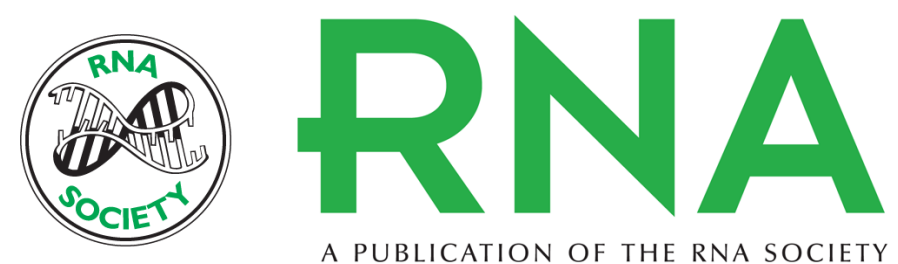

A PUBLICATION OF THE RNA SOCIETY

\title{
Discovery and investigation of the GAIT translational control system
}

\author{
Paul L. Fox
}

RNA 2015 21: 615-618
Open Access Freely available online through the RNA Open Access option.
Creative This article, published in $R N A$, is available under a Creative Commons License Commons (Attribution-NonCommercial 4.0 International), as described at License http://creativecommons.org/licenses/by-nc/4.0/. Email Alerting
Service 